TRANSACTIONS OF THE

AMERICAN MATHEMATICAL SOCIETY

Volume 360, Number 10, October 2008, Pages 5589-5602

S 0002-9947(08)04513-3

Article electronically published on May 22, 2008

\title{
DIMENSION OF ESCAPING GEODESICS
}

\author{
ZSUZSANNA GÖNYE
}

\begin{abstract}
Suppose $M=\mathbb{B} / G$ is a hyperbolic manifold. Consider the set of escaping geodesic rays $\gamma(t)$ originating at a fixed point $p$ of the manifold $M$, i.e. $\operatorname{dist}(\gamma(t), p) \rightarrow \infty$. We investigate those escaping geodesics which escape at the fastest possible rate, and find the Hausdorff dimension of the corresponding terminal points on the boundary of $\mathbb{B}$.

In dimension 2, for a geometrically infinite Fuchsian group, if the injectivity radius of $M=\mathbb{B} / G$ is bounded above and away from zero, then these points have full dimension. In dimension 3 , when $G$ is a geometrically infinite and topologically tame Kleinian group, if the injectivity radius of $M=\mathbb{B} / G$ is bounded away from zero, the dimension of these points is 2 , which is again maximal.
\end{abstract}

\section{INTRODUCTION}

Consider $G$, a discrete, torsion free group of isometries of the hyperbolic metric on the hyperbolic three ball $\mathbb{B}$; i.e. a Kleinian group. Passing to the quotient $\mathbb{B} / G$ by identification of the $G$-equivalent points we obtain the quotient space $M$, which is a manifold. Suppose this group $G$ is non-elementary, and denote its limit set by $\Lambda$.

A point $x$ on the boundary of the ball is a non-conical point if there is a geodesic ray ending at $x$ so that the projection of this geodesic will eventually leave any compact set and tend to the ideal boundary. Among these points there is a subset that escapes to the ideal boundary at the fastest possible rate; these are called deep points. The original definition of deep points is due to McMullen ([14]). A point is a deep point if there is a geodesic ray $\gamma:[0, \infty) \rightarrow C(\Lambda)$ in the convex hull parameterized by arclength and terminating at $x$, so that for some $\delta>0$

$$
\frac{\operatorname{dist}(\gamma(t), \partial C(\Lambda))}{t} \geq \delta
$$

for all $t$, i.e. the depth of $\gamma$ inside the convex hull of the limit set $\Lambda$ increases linearly with the hyperbolic length. (All the necessary definitions will be given later in the text.)

We can generalize this notation by taking any Lipschitz function $\phi(t):[1, \infty) \rightarrow$ $[1, \infty)$ with the property of $\lim _{t \rightarrow \infty} \phi(t)=\infty$. We fix a point $z_{0} \in M=\mathbb{B} / G$, and

Received by the editors November 29, 2005 and, in revised form, March 9, 2007.

2000 Mathematics Subject Classification. Primary 30F40, 28A78; Secondary 30F35.

Key words and phrases. Fuchsian groups, Kleinian groups, escaping geodesics, deep points, Hausdorff dimension.

(C)2008 American Mathematical Society Reverts to public domain 28 years from publication 
consider the set of geodesic rays starting at $z_{0}$ parameterized by the hyperbolic arclength. Define the set of geodesics in the convex core which escape at a rate $\phi$ as

$$
\Gamma_{\phi}^{C}=\left\{\gamma: \frac{1}{C} \leq \frac{\operatorname{dist}\left(\gamma(t), z_{0}\right)}{\phi(t)} \leq C\right\} .
$$

Let $\Lambda_{\phi}^{C}$ denote the terminating points of the geodesics in $\Gamma_{\phi}^{C}$, and let $\Lambda_{\phi}=\bigcup_{C} \Lambda_{\phi}^{C}$.

The main theorem of this paper is the following:

Theorem 1.1. Suppose $G$ is a geometrically infinite, topologically tame Kleinian group, $M=\mathbb{B} / G$ has injectivity radius bounded away from zero and there is a Green's function on $M$. Let $\phi(t):[1, \infty) \rightarrow[1, \infty)$ be a Lipschitz function satisfying $\lim _{t \rightarrow \infty} \phi(t)=\infty$; then the Hausdorff dimension of $\Lambda_{\phi}$ is 2 .

The relevant definitions will be given later in Sections 2 , 3 and 4 . The idea of the proof is as follows: we can find a positive harmonic function $u$ on the manifold $M$ which tends to 0 in the geometrically finite ends of $M$ (Lemma 4.1). Lifting $u$ up to the covering space $\mathbb{B}$, we get a hyperbolic harmonic function $U$ on the ball $\mathbb{B}$. This hyperbolic harmonic function is a Poisson integral of some positive measure $\mu$, which is supported on the limit set. Using this measure $\mu$, we construct a Bloch martingale $\left\{f_{n}\right\}$ on the dyadic squares $Q$ of length $2^{-n}$ by defining $f_{n}$ as

$$
f_{n}(x)=\frac{\mu(Q(x))}{m(Q(x))} .
$$

With the help of a technical lemma (Lemma 5.2) we can find a Cantor set, which has Hausdorff dimension two (Lemma 3.3), on which the martingale grows approximately at the same rate as the given Lipschitz function $\phi$, i.e.

$$
\frac{1}{C} \leq\left|\frac{f_{n}(Q)}{\phi(n)}\right| \leq C
$$

The martingale $f_{n}(x)$ for $x \in Q$ has bounded distance from the harmonic function $U$ on the top of the Carleson square drawn over Q (Lemma 4.2); therefore

$$
\frac{1}{C} \leq\left|\frac{U\left(z_{Q}\right)}{\phi(n)}\right| \leq C
$$

Finally, $U(z)$ gives the distance approximately from $\gamma(t)$ to the base point, which gives an estimate for $\operatorname{dist}(\gamma(t), \partial C(\Lambda))$ on manifolds specified in the main theorem.

An analogous theorem can also be given for Fuchsian groups:

Theorem 1.2. Suppose $G$ is a geometrically infinite Fuchsian group, $M=\mathbb{B} / G$ has injectivity radius bounded and bounded away from zero, and there is a Green's function on $M$. Let $\phi(t):[1, \infty) \rightarrow[1, \infty)$ be a Lipschitz function satisfying $\lim _{t \rightarrow \infty} \phi(t)=\infty$; then the Hausdorff dimension of $\Lambda_{\phi}$ is 1 .

Fernández and Melián in [9] extensively studied the size of the set of escaping geodesics starting at a point of the hyperbolic surface. A geodesic ray $\gamma(t)$ originating at $p$ is called escaping if $\lim _{t \rightarrow \infty} \operatorname{dist}(\gamma(t), p)=+\infty$. These rays may leave the convex core. It is known that if there is a Green's function on the surface, then the set of escaping geodesics has full measure. If there is no Green's function, then the Hausdorff dimension of the terminal points is still 1. 
Taking $\phi(t)=t$ in Theorems 1.1 and 1.2 we get the dimension of deep points in sets described in the theorem above.

Corollary 1.3. If $G$ is a geometrically infinite, topologically tame Kleinian group and $M=\mathbb{B} / G$ has injectivity radius bounded away from zero and has a Green's function, then the deep points have dimension 2 .

\section{Definitions And notation}

Let $M\left(\overline{\mathbb{R}}^{d}\right)$ denote the orientation preserving Möbius transformations in the $d$-dimensional extended space $\overline{\mathbb{R}}^{d}=\mathbb{R}^{d} \cup\{\infty\}$. The subgroup of $M\left(\overline{\mathbb{R}}^{d}\right)$ which preserves the upper half-plane $\mathbb{H}=\left\{x \in \overline{\mathbb{R}}^{d}: x_{d}>0\right\}$ or the unit ball $\mathbb{B}=$ $\left\{x \in \overline{\mathbb{R}}^{d}:|x|<1\right\}$ will be denoted by $M(\mathbb{H})$ and $M(\mathbb{B})$, respectively. A discrete group $G$ of $M(\mathbb{B})$ in dimension 3 is called a Kleinian group. A Fuchsian group is a Kleinian group that stabilizes a round disc on $\partial \mathbb{B}$, the sphere at infinity. In this paper we consider only non-elementary groups, that is, $G$ has no finite orbit in $\mathbb{H}^{3}=\left\{x \in \overline{\mathbb{R}}^{3}: x_{3}>0\right\}$.

If $G$ is a discrete subgroup of $M(\mathbb{B})$, the orbit $G(a)$ of any point $a \in \mathbb{B}$ can accumulate only on the boundary of $\mathbb{B}$. So we call a point $x \in S=\partial \mathbb{B}$ a limit point, if there is an orbit $G(a)$ accumulating at $x$. The limit set is the set of limit points and is denoted by $\Lambda(G)$ or simply by $\Lambda$. The complementary set $S \backslash \Lambda$ of $\Lambda$ is called the ordinary set, and is denoted by $\Omega([3])$.

Let $G$ be a Kleinian group. Then the quotient space $\Omega / G$, which is obtained from the ordinary set of $G$ by identifying equivalent points under the mappings of $G$, is a marked (possibly disconnected) Riemann surface ([13]). If $\Omega / G$ is a finite marked Riemann surface (i.e. a finite union of compact surfaces, each with at most a finite number of punctures), then $G$ is called analytically finite. The Ahlfors finiteness theorem shows that $G$ is analytically finite if it is finitely generated.

A Möbius group $G$ is called geometrically finite if some convex fundamental polyhedron has finitely many faces. In dimensions 2 and 3 the standard definition of geometric finiteness is that the Dirichlet region must have finitely many faces. It is known that this criterion implies that every Dirichlet region and every convex fundamental polyhedron has finitely many faces ([3], [15]). Moreover, geometric finiteness implies that the group is finitely generated, and therefore analytically finite.

The convex hull of $\Lambda \subset S=\partial \mathbb{B}$, denoted by $C(\Lambda)$, is the smallest convex subset of $\mathbb{B}$ containing all geodesics with both endpoints in $\Lambda$. The convex core of a hyperbolic manifold $M=\mathbb{B} / G$ is given by the quotient $C(\Lambda) / G$ and denoted by $C(M)$. For $x \in M$ the injectivity radius, $\operatorname{inj}(x)$, is half the distance between the two closest distinct lifts of $x$ to $\mathbb{B}$. In the theorem we assume that the injectivity radius is bounded away from zero uniformly on $M$, which in dimensions 2 and 3 implies that $G$ has no parabolic elements.

A Kleinian group is called topologically tame, if the corresponding quotient manifold $M=\mathbb{B} / G$ is homeomorphic to the interior of a compact 3-manifold with boundary. This implies that the convex core $C(M)$ consists of a compact piece and a finite number of ends $E_{j}$, which are topologically equivalent to $S \times \mathbb{R}_{+}$for some compact surface $S$. D. Calegari and D. Gabai in [6] and I. Agol in [1] proved the Marden conjecture, where all complete hyperbolic 3-manifolds with finitely generated fundamental group are topologically tame. We note here that in 7] Canary 
showed that topological tameness is equivalent to analytical tameness in dimension 3. Moreover, if $G$ is topologically tame, then there is an upper bound for the injectivity radius inside the convex core.

In the Introduction we gave the definition of a deep point defined by a geodesic ray in the convex hull of $\Lambda$. An equivalent definition can also be given on the quotient manifold, as in [4. A point $x \in \Lambda$ is deep if the geodesic ray $\gamma$ ending at $x$ satisfies

$$
\frac{\operatorname{dist}(\tilde{\gamma}(t), M \backslash C(M))}{t} \geq \delta>0
$$

for all $t \geq t_{0}$, where $\tilde{\gamma}$ denotes the curve on the quotient space which corresponds to $\gamma$.

\section{Dyadic martingale and Hausdorff Dimension}

An $n$th generation dyadic cube in $\mathbb{R}^{d}$ is

$$
Q_{n}=\left\{x=\left(x_{1}, x_{2}, \ldots, x_{d}\right): a_{i} \leq x_{i}<a_{i}+2^{-n}, 1 \leq i \leq d\right\}
$$

where $a=\left(a_{1}, a_{2}, \ldots, a_{d}\right)$, the corner of the cube, has coordinates in the form $a_{i}=\frac{m_{i}}{2^{n}}$ with an integer $m_{i}$. The collection of these dyadic cubes is denoted by $\mathcal{D}_{n}$. For any given point $x \in \mathbb{R}^{d}$ let $Q_{n}(x)$ denote that unique $n$th generation dyadic cube from $\mathcal{D}_{n}$ which contains the point $x$, and let $\left|Q_{n}\right|$ denote the side-length of $Q_{n}$. The $m$ th generation descendants of $Q_{n}$ are the dyadic sub-cubes of $Q_{n}$ with side-length of $2^{-m}\left|Q_{n}\right|$. There are $2^{m d}$ of them.

Suppose $Q_{0}$ is a unit cube in $\mathbb{R}^{d}$. Then a sequence of functions $\left\{f_{n}\right\}_{n=0}^{\infty}$ is said to be a dyadic martingale on $Q_{0}$ if

(1) $f_{n}$ is measurable on each $Q_{n} \in \mathcal{D}$,

(2) $\frac{1}{\left|Q_{n}\right|} \int_{Q_{n}} f_{n}<\infty$

(3) $\frac{1}{\left|Q_{m}\right|} \int_{Q_{m}} f_{n}=f_{m}$ for all $m<n$.

In addition to this usual definition, we also require that $f_{n}$ must be constant on the $n$th generation dyadic cubes. Since in this paper we use only dyadic martingales, so we often omit the "dyadic" attribute.

There is a standard way to construct a martingale from a finite measure. If a finite measure $\mu$ is given on $Q_{0}$, then the functions

$$
f_{n}(x)=\frac{\mu\left(Q_{n}(x)\right)}{\left|Q_{n}(x)\right|_{d}}
$$

define a dyadic martingale, where $\left|Q_{n}(x)\right|_{d}$ (or just $\left|Q_{n}(x)\right|$ if the notation is clear from the text) denotes the $d$-dimensional Lebesgue measure of $Q_{n}(x)$. We define the martingale differences as $\Delta f_{n}(x)=f_{n+1}(x)-f_{n}(x)$ and the martingale square function as

$$
S_{f}(x)=\left(\sum_{n=1}^{\infty}\left\|\chi_{Q_{n}(x)} \Delta f_{n}\right\|_{\infty}^{2}\right)^{1 / 2} .
$$

A martingale is called Bloch if $\sup _{n}\left\|\Delta f_{n}\right\|_{\infty}<\infty$. If $\left\{f_{n}\right\}$ is an $L^{1}$-bounded martingale, then $f_{n}$ converges a.e. to a function $f$ with $\|f\|_{1}<\infty$. For more results on the convergence of martingales, you may see [11]. We will need the following two estimates for dyadic martingales. 
Lemma 3.1. Let $f_{n}$ be a dyadic martingale on $Q_{0} \subset \mathbb{R}^{d}$ with limit function $f$. Suppose $\left\|S_{f}\right\|_{\infty}<\infty$. Then for $\lambda \geq 0$,

$$
\left|x \in Q_{0}: f(x)-f_{0}(x) \geq \lambda\right| \leq \exp \left(-\frac{\lambda^{2}}{2\left\|S_{f}\right\|_{\infty}^{2}}\right)
$$

The proof of this lemma is due to Herman Rubin and can be found in the paper of Chang-Wilson-Wolff [8, Theorem 3.1].

Lemma 3.2. Suppose $\mu$ is a probability measure on $X$. Suppose $F$ is a measurable, real valued function on $X$ so that $\int_{X} F d \mu=0$ and $\|F\|_{4} \leq B\|F\|_{2}$. Then

$$
\mu\left(\left\{x: F(x) \leq-\frac{1}{\sqrt{8} B^{2}}\|F\|_{2}\right\}\right) \geq \frac{1}{64 B^{12}} .
$$

The proof is given in [4].

Suppose $\phi$ is an increasing, continuous function from $[0, \infty)$ to itself such that $\phi(0)=0$. For a given set $E$ we define the Hausdorff content as

$$
\mathcal{H}_{\infty}^{\phi}(E)=\inf \left\{\sum \phi\left(r_{j}\right): E \subset \cup_{j} D\left(x_{j}, r_{j}\right)\right\},
$$

where $D\left(x_{j}, r_{j}\right)$ denotes a ball of radius $r_{j}$ centered at $x_{j}$. Especially, if $\phi(t)=t^{\alpha}$ we denote $\mathcal{H}_{\infty}^{\phi}$ by $\mathcal{H}_{\infty}^{\alpha}$. The Hausdorff dimension of a set $E$ is

$$
\operatorname{dim}_{\mathrm{H}}(E)=\inf \left\{\alpha: \mathcal{H}_{\infty}^{\alpha}(E)=0\right\} .
$$

For more details and examples on Hausdorff dimension you may see [5].

In the proof of our theorem we will also need the following lemma.

Lemma 3.3. Suppose $E_{n}$ is a union of closed dyadic cubes of generation $k_{n}$ so that $E_{0} \supset E_{1} \supset E_{2} \supset \ldots$ and there are constants $N$ and $\epsilon$ with

(1) $\left|k_{n}-k_{n+1}\right|=N$ for all $n$.

(2) If $Q \in E_{n}$ is generation $k_{n}$, then $\left|E_{n+1} \cap Q\right|_{d} \geq \epsilon|Q|_{d}$.

If $E=\bigcap_{n} E_{n}$, then $\operatorname{dim}_{\mathrm{H}}(E) \geq d-C(N, \epsilon)$, where $C(N, \epsilon) \rightarrow 0$ whenever $\epsilon>0$ is fixed and $N \rightarrow \infty$.

The proof of this lemma can be found in [10, 12] and [16].

\section{The HYPERBOLIC SPACE AND HARMONIC FUNCTIONS}

The unit ball $\mathbb{B}$ in $\mathbb{R}^{n}$ is the disc model for the $n$-dimensional hyperbolic space equipped with the hyperbolic metric

$$
d \rho=\frac{2|d x|}{1-|x|^{2}} .
$$

An alternative model of the hyperbolic $n$-space is the upper half-plane $\mathbb{H}=\{x=$ $\left.\left(x_{1}, x_{2}, \ldots, x_{n}\right): x_{n}>0\right\} \subset \mathbb{R}^{n}$ equipped with the metric

$$
d \rho=\frac{|d x|}{x_{n}} .
$$

Using the hyperbolic metric defined in $\mathbb{B}=\left\{x \in \mathbb{R}^{n}:|x|<1\right\}$ by (4.1) we may construct the hyperbolic volume and area element, the normal derivative and 
gradient in the hyperbolic ball as

$$
\begin{aligned}
d V_{H} & =\frac{2^{n} d x_{1} d x_{2} \cdots d x_{n}}{\left(1-|x|^{2}\right)^{n}}, \\
d \sigma_{H} & =\frac{2^{n-1} d \sigma}{\left(1-|x|^{2}\right)^{n-1}}, \\
\frac{\partial v}{\partial n_{H}} & =\frac{1-|x|^{2}}{2} \frac{\partial v}{\partial n}, \\
\nabla_{H} u & =\frac{1-|x|^{2}}{2} \nabla u .
\end{aligned}
$$

On the upper half-plane $\mathbb{H}=\left\{x=\left(x_{1}, \ldots, x_{n}\right) \in \mathbb{R}^{n}, x_{n}>0\right\}$ these are

$$
\begin{aligned}
d V_{H} & =\frac{d x_{1} \cdots d x_{n}}{x_{n}^{n}}, \\
d \sigma_{H} & =\frac{d \sigma}{x_{n}^{n-1}}, \\
\frac{\partial v}{\partial n_{H}} & =x_{n} \frac{\partial v}{\partial n}, \\
\nabla_{H} u & =x_{n} \nabla u,
\end{aligned}
$$

respectively. A more detailed description can be found in [2] and [15].

The hyperbolic Laplace-Beltrami operator for the unit ball $\mathbb{B} \subset \mathbb{R}^{n}$ is given by

$$
\Delta_{H}=\frac{\left(1-r^{2}\right)^{2}}{4}\left[\Delta+\frac{2(n-2) r}{1-r^{2}} \frac{\partial}{\partial r}\right]
$$

where $r=|x|$. On the upper half-plane this is

$$
\Delta_{H}=x_{n}^{2}\left[\Delta-\frac{n-2}{x_{n}} \frac{\partial}{\partial x_{n}}\right] .
$$

A function $f$ is called hyperbolically harmonic if it satisfies the hyperbolic Laplace equation, $\Delta_{H} f=0$.

We define the Green's function on a quotient manifold $M$ as follows. $F$ is a Green's function on $M$ with a pole at the projection of a point $a$, if there exists a function $f: \mathbb{B} \backslash\{G(a)\} \rightarrow \mathbb{R}$ such that the projection of $f$ is $F$ and the following are true for $f$ :

- $f$ is a hyperbolic harmonic function on $\mathbb{B} \backslash\{G(a)\}$,

- $f \circ g=f$ for all $g \in G$,

- $\lim _{z \rightarrow a}\left(f(z)-\frac{1}{z-a}\right)$ exists, i.e. $f$ has singularity $\frac{1}{z-a}$ at the point $a$,

- $f$ is the smallest positive function with these properties.

The hyperbolic version of Green's formula is

$$
\int_{D}\left(u \Delta_{H} v-v \Delta_{H} u\right) d V_{H}=\int_{\partial D}\left(u \frac{\partial v}{\partial n_{H}}-v \frac{\partial u}{\partial n_{H}}\right) d \sigma_{H} .
$$

We will need the following existence theorem, which was proven in [4].

Lemma 4.1. Suppose $G$ is topologically tame, geometrically infinite, $M=\mathbb{B} / G$ has an injectivity radius bounded below by $\epsilon>0$ and Green's function $G(w, z)$ exists on $M$. Then there exists a positive harmonic function $U$ on $M$ such that

$$
\sup _{z \in M}|\nabla U(z)| \leq 1
$$


and $U$ tends to zero in the geometrically finite ends of $M$. If, in addition, $G$ is topologically tame, then for any $a_{0}>0$ there are constants $a_{1}$ and $a_{2}$ so that

$$
\int_{B\left(z, a_{1}\right)}|\nabla U|^{2} d V \geq a_{2}
$$

for every $z$ such that $\operatorname{dist}(z, C(M)) \leq a_{0}$. Moreover, $U(z)$ tends to $+\infty$ in the geometrically infinite ends as $\operatorname{dist}(z, \partial C(M)) \rightarrow \infty$.

If $Q$ is a cube in $\mathbb{R}^{n}$, then $\hat{Q}=Q \times[0, \ell(Q)]$ is called the Carleson cube in $\mathbb{R}_{+}^{n+1}$ with base $Q$, and let $z_{Q}$ denote the center of $\hat{Q}$.

Lemma 4.2. Suppose $U$ on $\mathbb{R}_{+}^{n+1}$ is the hyperbolic Poisson integral of the positive measure $\mu$ and satisfies $\left|\nabla_{H} U(z)\right| \leq 1$. For a square $Q \in \mathbb{R}^{n}$, let $Q_{t}=\{(\underline{x}, t)$ : $\underline{x} \in Q\}$. Then there is an $A<\infty$ so that

$$
\left|U\left(z_{Q}\right)-\frac{1}{|Q|} \int_{Q} U(\underline{x}, t) d \underline{x}\right| \leq A,
$$

for any $0<t \leq \ell(Q)$, where $\ell(Q)$ denotes the side-length of $Q$, and

$$
\left|U\left(z_{Q}\right)-\frac{1}{|Q|} \int_{Q} d \mu\right| \leq A .
$$

The proof of this lemma in dimension $n=2$ was given in [4, and it can be proven in higher dimension on a similar way as in 10 .

\section{TWO LEMMAS ON MARTINGALES}

Define a martingale on the dyadic cubes using the positive measure $\mu$ described in Lemma 4.1 defined by equation (3.1). According to Lemma 4.2, there exists a constant $A$ so that $\left|U\left(z_{Q_{n}(x)}\right)-f_{n}(x)\right| \leq A$. To prove our main theorem we will need the following two lemmas for dyadic martingales.

Lemma 5.1. Suppose $\mu$ is a positive measure on the cube $[0,1]^{d}, d \geq 1$, so that the corresponding dyadic martingale defined by $f_{n}(x)=\frac{\mu\left(Q_{n}(x)\right)}{\left|Q_{n}(x)\right|}$ is Bloch and $\frac{1}{\left|Q_{n}\right|}\left\|\Delta f_{n}\right\|_{2}^{2} \geq \delta>0$ whenever $f_{n}(x) \geq 1$ on $Q_{n}(x)$. We claim that there is an $\epsilon>0$ and $M<\infty$ so that for any sufficiently large $n$, there is a constant $C$ for which the following holds. Let $Q$ be any dyadic cube, and let $f_{Q}$ denote the function in the martingale defined by $Q$, i.e. $f_{Q}=\frac{\mu(Q)}{|Q|}$ on $Q$. Suppose $f_{Q} \geq C$. Then among the $2^{d n} n$th generation descendants of $Q$, at least $\epsilon 2^{d n}$ satisfy $M n \geq f_{Q^{\prime}}-f_{Q} \geq 1$, and at least $\epsilon 2^{d n}$ satisfy $-M n \leq f_{Q^{\prime}}-f_{Q} \leq-1$.

Proof. Suppose $\sup _{n}\left\|\Delta f_{n}\right\|_{\infty}=L<\infty$ and $\frac{1}{Q_{n}}\left\|\Delta f_{n}\right\|_{2}^{2} \geq \delta>0$ whenever $f_{n}(x) \geq 1$, and fix an $\epsilon$ with $0<\epsilon \leq \min \left\{\frac{\delta^{6}}{2^{16} L^{12}}, 1\right\}$. By an appropriate scaling we may assume that $|Q|=1$. Then the martingale square function for the sequence $\left\{f_{0}, f_{1}, \ldots, f_{n}\right\}$ is

$$
S_{f}(x)=\left(\sum_{j=0}^{n-1}\left\|\chi_{Q_{j}(x)} \Delta f_{j}\right\|_{\infty}^{2}\right)^{1 / 2} \leq \sqrt{n} L .
$$


Let $F=\Delta f_{0}+\ldots+\Delta f_{n-1}$, and suppose that $n>\frac{64 L^{4}}{\delta^{3}}$ and that $f_{Q} \geq 1+n L=C$. Then $f_{j} \geq 1$ for all $0 \leq j \leq n-1$, so $\left\|\Delta f_{j}\right\|_{2}^{2} \geq \delta$ for all $0 \leq j \leq n-1$. The system $\left\{\Delta f_{j}\right\}_{j=0}^{n-1}$ is orthogonal, therefore

$$
n L^{2} \geq\|F\|_{2}^{2}=\sum_{j=0}^{n-1}\left\|\Delta f_{j}\right\|_{2}^{2} \geq n \delta .
$$

Let $\lambda(t)=|\{x:|F(x)|>t\}|$ define the distribution function of $|F|$. Then

$$
\int|F|^{p}=p \int_{0}^{\infty} t^{p-1} \lambda(t) d t
$$

and by Lemma 3.1, $\lambda(t) \leq e^{-\frac{t^{2}}{2\left\|S_{F}\right\|_{\infty}^{2}}} \leq e^{-\frac{t^{2}}{2 L^{2} n}}$. Therefore

$$
\begin{aligned}
\|F\|_{4}^{4} & =\int|F|^{4}=4 \int_{0}^{\infty} t^{3} \lambda(t) d t \\
& \leq 4 \int_{0}^{\infty} t^{3} e^{-\frac{t^{2}}{2 L^{2} n}} d t \\
& =8 L^{4} n^{2} \int_{0}^{\infty} y e^{-y} d y \\
& =8 L^{4} n^{2} .
\end{aligned}
$$

Hence $\|F\|_{4} \leq \sqrt[4]{8} L \sqrt{n}=B \delta \sqrt{n} \leq B\|F\|_{2}$ with the constant $B=\frac{\sqrt[4]{8} L}{\sqrt{\delta}}$. Now we can apply Lemma 3.2 so

$$
\mu\left(\left\{x \in Q: F(x) \leq-\frac{1}{\sqrt{8} B^{2}}\|F\|_{2}\right\}\right) \geq \frac{1}{64 B^{12}} .
$$

Using the fact that $\|F\|_{2} \geq \sqrt{n \delta}$ and the assumptions that $\sqrt{n}>\frac{8 L^{2}}{\sqrt{\delta}}=\frac{\sqrt{8}}{\sqrt{\delta}} B^{2}$ we get that

$$
\begin{aligned}
\mu(\{x \in Q: F(x) \leq-1\}) & \geq \mu\left(\left\{x \in Q: F(x) \leq-\frac{1}{\sqrt{8} B^{2}} \sqrt{n \delta}\right\}\right) \\
& \geq \mu\left(\left\{x \in Q: F(x) \leq-\frac{1}{\sqrt{8} B^{2}}\|F\|_{2}\right\}\right) \\
& \geq \frac{1}{64 B^{12}} \\
& =\frac{\delta^{6}}{2^{15} L^{12}} \\
& \geq 2 \epsilon .
\end{aligned}
$$

Switching $F$ with $-F$, with the same assumptions, we get

$$
\mu(\{x \in Q: F(x) \geq 1\}) \geq 2 \epsilon .
$$


Next, consider the following subsequence $\left\{f_{0}, \ldots, f_{n}\right\}$. By Lemma 3.1, for a positive constant $M \geq L \sqrt{-2 \ln \epsilon}$,

$$
\begin{aligned}
\left|\left\{x \in Q: f_{n}(x)-f_{0}(x) \geq n M\right\}\right| & \leq \exp \left(\frac{-n^{2} M^{2}}{2\left\|S_{f}\right\|_{\infty}^{2}}\right) \\
& \leq \exp \left(\frac{-n M^{2}}{2 L^{2}}\right) \\
& \leq\left(\exp \left(-\frac{M^{2}}{2 L^{2}}\right)\right)^{n} \\
& \leq \epsilon
\end{aligned}
$$

for every $n \geq 1$ and $\epsilon<1$. Repeating this argument for $\left\{-f_{0}, \ldots,-f_{n}\right\}$ we get that

$$
\left|\left\{x \in Q: f_{n}(x)-f_{0}(x) \leq-n M\right\}\right| \leq \epsilon .
$$

Therefore, for every sufficiently large $n$ there is a constant $C=1+n L$ so that if $f_{Q} \geq C$, then

$$
\mu\left(\left\{x \in Q: 1 \leq F(x)=f_{n}(x)-f_{0}(x) \leq n M\right\}\right) \geq \epsilon
$$

and

$$
\mu\left(\left\{x \in Q:-1 \geq F(x)=f_{n}(x)-f_{0}(x) \geq-n M\right\}\right) \geq \epsilon .
$$

In other words, among all of the $2^{d n} n$th generation descendants of $Q$, at least $\epsilon 2^{d n}$ satisfy the inequality $M n \geq f_{Q^{\prime}}-f_{Q} \geq 1$, and at least $\epsilon 2^{d n}$ of them satisfy $-M n \leq f_{Q^{\prime}}-f_{Q} \leq-1$.

Lemma 5.2. Suppose $G$ is a topologically tame and geometrically infinite Kleinian group, so that $M=\mathbb{B} / G$ has injectivity radius bounded below by some positive epsilon and there exists a Green's function on $M$. Let $U$ be a positive harmonic function on $M$ for which $\sup _{z \in M}\left|\nabla_{H} U(z)\right| \leq 1$, and let $\left\{f_{m}\right\}$ denote the corresponding martingale as defined by (3.1). Then this martingale $\left\{f_{m}\right\}$ has bounded differences away from zero in the $L_{2}$ norm, whenever its value is larger than some fixed constant $C$.

Proof. Suppose $f_{m}>C$ on the dyadic cube $Q_{m}$. From Lemma 4.2 we know that $\left|U\left(z_{Q}\right)-f_{m}\right| \leq A$, where $z_{Q}$ denotes the center of the Carleson square in $\mathbb{R}_{+}^{n+1}$ with base $Q_{m}$. Since $G$ is topologically tame and $\operatorname{inj}(z) \geq \epsilon>0$, the convex core $C(M)$ can be written as a compact part and a finite number of ends $E_{j}$, each of which is topologically equivalent to $S \times \mathbb{R}^{+}$for some compact surface $S$ ([7]). We may suppose that we are already in such an end.

First, we will show that for the given constant $A$, there exists a constant $L$, so that for all $v \in C(M)$ with $U(v) \geq C$ we can find another point $w$ with $\rho(v, w) \leq L$ and $|U(v)-U(w)| \geq 6 A$. Lemma 4.1 says that there exist $r$ and $a$ so that

$$
\int_{B(z, r)}|\nabla U|^{2} d V \geq a
$$

for every $z \in C(M)$. Consider a geodesic ray on $M$ originating at the point $v$ and going to infinity in the convex core. We may put disjoint balls of radius $r$ along this geodesic, say $N$ balls, and denote $w$ the endpoint, so $\rho(v, w)=2 r N$. Cut $E_{j}$ at $v$ and at $w$, and call these surfaces $\Sigma_{1}$ and $\Sigma_{2}$, respectively, and let $T$ denote 
the part of $E_{j}$ between these cuts. We may also assume that $U(v)=0$. Green's Theorem says that

$$
\int_{T} f \Delta g-g \Delta f d V_{H}=\int_{\Sigma_{1} \cup \Sigma_{2}} f \frac{\partial g}{\partial n}-g \frac{\partial f}{\partial n} d \sigma .
$$

Let $f=1$ and $g=U^{2}$; then

$$
\int_{T} \Delta\left(U^{2}\right) d V=\int_{\Sigma_{1} \cup \Sigma_{2}} \frac{\partial\left(U^{2}\right)}{\partial n} d \sigma .
$$

By elementary calculations we get that

$$
\int_{T}|\nabla U|^{2} d V=\int_{\Sigma_{1} \cup \Sigma_{2}} U \frac{\partial U}{\partial n} d \sigma .
$$

Using Lemma 4.1, we can estimate the left-hand side of (5.13) by

$$
\int_{T}|\nabla U|^{2} \geq N a
$$

For the estimation of the right-hand side of (5.13) we can use that $|\nabla U| \leq 1$, so

$$
\begin{aligned}
& \int_{\Sigma_{1}} U \frac{\partial U}{\partial n} d \sigma \leq \operatorname{diam}\left(\Sigma_{1}\right) \operatorname{area}\left(\Sigma_{1}\right), \\
& \int_{\Sigma_{2}} U \frac{\partial U}{\partial n} d \sigma \leq\left(U(w)+\operatorname{diam}\left(\Sigma_{2}\right)\right) \operatorname{area}\left(\Sigma_{2}\right) .
\end{aligned}
$$

Since $E=S \times \mathbb{R}^{+}, \operatorname{diam}\left(\Sigma_{i}\right) \leq D$ and area $\left(\Sigma_{i}\right) \leq S$ along the entire end $E_{j}$. Using the estimations (5.14) and (5.15) in (5.13), we get that

$$
N a \leq D S+(U(w)+D) S,
$$

and so

$$
\frac{N a}{S}-2 D \leq U(w)
$$

Therefore, we can choose a uniform $N$ large enough so that $|U(v)-U(w)| \geq 6 A$ and let $L=2 r N$.

Next, we show that there is a point $w$ such that $\left|U\left(z_{Q}\right)-U(w)\right| \geq 3 A$ while $\rho\left(z_{Q}, w\right) \leq 3 L$. We start at the point $z_{Q}$ on the Carleson square and go straight down toward the boundary by hyperbolic distance $2 L$; we call this point $v$. As we have just shown above, there exists a $w$ such that $\rho(v, w) \leq L$ while $|U(v)-U(w)| \geq$ $6 A$, which means that either $\left|U\left(z_{Q}\right)-U(v)\right| \geq 3 A$ or $\left|U\left(z_{Q}\right)-U(w)\right| \geq 3 A$. Assume the latter is true.

Finally, we show that there is a subfamily $\left\{f_{m_{i}}\right\}$ in the original martingale sequence with bounded differences away from zero in $L_{2}$-norm while $m_{i+1}-m_{i} \leq 3 L$ for all $i$. From Lemma 4.2 $\left|U\left(z_{Q}\right)-f_{m}\right| \leq A$ and we can find a $w$ such that $\left|U\left(z_{Q}\right)-U(w)\right| \geq 3 A$, but $\rho\left(z_{Q}, w\right) \leq 3 L$. We may also assume that $w$ is in the middle of a Carleson square, since $|\nabla U| \leq 1$. This Carleson square is different from the original $Q_{m}$, call it $Q_{m^{\prime}}$, and let $f_{m^{\prime}}$ be the martingale function determined by the size of this square. Then from Lemma 4.2 we have that $\left|U(w)-f_{m^{\prime}}\right| \leq A$ and $\left|U\left(z_{Q}\right)-f_{m}\right| \leq A$, while $\left|U\left(z_{Q}\right)-U(w)\right| \geq 3 A$. So $\left|f_{m}-f_{m^{\prime}}\right| \geq A$ on $Q_{m^{\prime}}$, while $\left|m-m^{\prime}\right| \leq 3 L$. Therefore

$$
\frac{1}{\left|Q_{m}\right|} \int_{Q_{m}}\left|f_{m}-f_{m^{\prime}}\right|^{2} d x \geq \frac{1}{\left|Q_{m}\right|} A^{2} \frac{\left|Q_{m}\right|}{2^{3 L}}=\delta>0 .
$$




\section{The PROOF OF THE THEOREM}

Suppose $G$ is a topologically tame, geometrically infinite Kleinian group and the quotient manifold $M=\mathbb{B} / G$ has injectivity radius bounded away from zero. This implies that $\mathrm{G}$ has no parabolic elements. Suppose $\phi(t):[1, \infty) \rightarrow[1, \infty)$ is a Lipschitz function, i.e.

$$
|\phi(s)-\phi(t)| \leq B|s-t|
$$

for some $B<\infty$, and satisfies $\lim _{t \rightarrow \infty} \phi(t)=\infty$. Fix a point $z_{0} \in M$ and consider the set of geodesic rays starting at $z_{0}$ parameterized by hyperbolic arclength. Define the set of geodesics in the convex core which escape at rate $\phi$ as

$$
\Gamma_{\phi}^{C}=\left\{\gamma: C^{-1} \leq \frac{\operatorname{dist}\left(\gamma(t), z_{0}\right)}{\phi(t)} \leq C\right\}
$$

Let $\Lambda_{\phi}^{C}$ denote the terminal points of these geodesics, and let $\Lambda_{\phi}=\bigcup_{C} \Lambda_{\phi}^{C}$.

Theorem 6.1. Suppose $G$ is a geometrically infinite, topologically tame Kleinian group and $M=\mathbb{B} / G$ has injectivity radius bounded away from zero and there is a Green's function on $M$. Let $\phi(t):[1, \infty) \rightarrow[1, \infty)$ be a Lipschitz function satisfying $\lim _{t \rightarrow \infty} \phi(t)=\infty$; then $\operatorname{dim}_{\mathrm{H}}\left(\Lambda_{\phi}\right)=2$.

The analogous theorem for Fuchsian groups:

Theorem 6.2. Suppose $G$ is a geometrically infinite Fuchsian group, $M=\mathbb{B} / G$ has bounded injectivity radius which is also bounded away from zero and there is a Green's function on $M$. Let $\phi(t):[1, \infty) \rightarrow[1, \infty)$ be a Lipschitz function satisfying $\lim _{t \rightarrow \infty} \phi(t)=\infty$; then $\operatorname{dim}_{\mathrm{H}}\left(\Lambda_{\phi}\right)=1$.

Proof of Theorem 6.1. By Lemma 4.1 there exists a positive harmonic function $U$ on $M$ with $\sup _{z \in M}|\nabla U(z)| \leq 1$ and $U$ tends to zero in the geometrically finite ends of $M$. This $U$ lifts to a hyperbolic harmonic function (which we will also call $U$ ) on $\mathbb{B}$, and this function is a Poisson integral of some positive measure $\mu$ supported on the limit set. Consider the corresponding dyadic Bloch martingale $\left\{f_{Q}(x)=\frac{\mu(Q(x))}{|Q(x)|}\right\}_{Q \in \mathcal{D}}$.

Using Lemma 5.2 we may pass to a subsequence of $\left\{f_{Q}\right\}$ for which the martingale differences are bounded away from zero whenever the value of the martingale is not less than a constant $C$. Notice that even if we work with this subsequence we can still use Lemma 5.1 because there are upper and lower bounds for the number of generations we skip. To simplify our indexes we will suppose that $\left\{f_{Q}\right\}$ itself is a Bloch martingale with differences bounded away from zero whenever the value is not less than $C$.

Using Lemma 5.1 we can create a Cantor set $\left\{E_{l}\right\}$ of nested dyadic cubes, where the dyadic martingale is comparable to the function $\phi$. As in Lemma 5.1 find appropriate $\epsilon>0, M<\infty$, and fix a sufficiently large $N$ and the corresponding constant $C$. Since $U$ tends to infinity on the geometrically infinite ends we may suppose that $f_{Q} \geq C$, except maybe for finitely many generations of cubes.

First, notice that we may suppose that the function $\phi$ is Lipschitz with a Lipschitz constant $\frac{1}{N}$, i.e. $|\phi(x)-\phi(y)| \leq \frac{1}{N}|x-y|$. In case $|\phi(x)-\phi(y)| \leq B|x-y|$ with a larger constant $B$ than $\frac{1}{N}$, we can rescale our function by choosing $\Phi(x)=\frac{1}{B N} \phi(x)$. 
Define $E_{0}$ as the collection of those largest cubes $Q$ where $f_{Q} \geq C$ and let $k_{0}$ be the number which denotes the generation of these cubes. Then there is a positive constant $D$ so that $\left|f_{k_{0}}(Q)-\phi\left(k_{0}\right)\right| \leq D$ on all $Q \in E_{0}$. We may also assume that $D \geq M N$. We define the sets $\left\{E_{n}\right\}$ inductively as follows. Suppose we already have the set $E_{n}$ defined, and the quotient (or the difference) of $f_{k_{l}}$ and $\phi\left(k_{l}\right)$ is bounded on all the previous sets, say $\frac{1}{D} \leq\left|f_{k_{l}}-\phi\left(k_{l}\right)\right| \leq D$ for all $l \leq n$. Let $k_{n+1}=k_{n}+N$. Since $\phi$ is a Lipschitz function with constant $\frac{1}{N}$, so $\phi\left(k_{n}\right)-1 \leq \phi\left(k_{n+1}\right) \leq \phi\left(k_{n}\right)+1$. To choose the next generation of cubes, for each $Q \in E_{n}$ we compare $f_{k_{n}}(Q)$ to $\phi\left(k_{n+1}\right)$ :

If $f_{k_{n}}(Q)<\phi\left(k_{n+1}\right)$, then choose those $N$ th generation descendants $Q^{\prime}$ of $Q$ for which $M N \geq f_{k_{n+1}}-f_{k_{n}} \geq 1$. According to Lemma 5.1 there are at least $\epsilon 2^{d N}$ of them, and then $f_{k_{n+1}}\left(Q^{\prime}\right)=f_{k_{n}}+a$, where $a \in[1, M N]$. Therefore, $\left|f_{k_{n+1}}\left(Q^{\prime}\right)-\phi\left(k_{n+1}\right)\right| \leq D$ because

$$
f_{k_{n+1}}-\phi\left(k_{n+1}\right)=f_{k_{n}}+a-\phi\left(k_{n+1}\right)<a \leq M N \leq D
$$

and

$$
\begin{aligned}
\phi\left(k_{n+1}\right)-f_{k_{n+1}} & =\phi\left(k_{n+1}\right)-f_{k_{n}}-a \\
& \leq \phi\left(k_{n}\right)-f_{k_{n}}+1-a \\
& \leq D+1-a \\
& \leq D .
\end{aligned}
$$

If $f_{k_{n}}(Q) \geq \phi\left(k_{n+1}\right)$, then choose those $N$ th generation descendants $Q^{\prime}$ of $Q$ for which $-M N \leq f_{k_{n+1}}-f_{k_{n}} \leq-1$. From Lemma 5.1] we know that there are at least $\epsilon 2^{d N}$ of them, and then $f_{k_{n}}\left(Q^{\prime}\right)=f_{k_{n}}-a$, where $a \in[1, M N]$. Therefore $\left|f_{k_{n+1}}\left(Q^{\prime}\right)-\phi\left(k_{n+1}\right)\right| \leq D$, because

$$
f_{k_{n+1}}-\phi\left(k_{n+1}\right)=f_{k_{n}}-a-\phi\left(k_{n+1}\right)>-a \geq-M N \geq-D
$$

and

$$
\begin{aligned}
\phi\left(k_{n+1}\right)-f_{k_{n+1}} & =\phi\left(k_{n+1}\right)-f_{k_{n}}+a \\
& \geq \phi\left(k_{n}\right)-f_{k_{n}}-1+a \\
& \geq-D+a-1 \\
& \geq-D .
\end{aligned}
$$

So we define the next imbedded set $E_{n+1}$ by

$$
E_{n+1}=\bigcup_{Q \in E_{n}}\{\text { all the chosen descendants of } Q\} \text {. }
$$

Then $E_{n+1} \subset E_{n}$, and for all $Q^{\prime} \in E_{n+1}$ we have $\left|f_{k_{n+1}}\left(Q^{\prime}\right)-\phi\left(k_{n+1}\right)\right| \leq D$; moreover,

$$
\left|E_{n+1} \cap Q\right|_{d} \geq \epsilon 2^{d N} \frac{|Q|_{d}}{2^{d N}}=\epsilon|Q|_{d}
$$

for all $Q \in E_{n}$. Since $\lim _{t \rightarrow \infty} \phi(t)=\infty$, the inequality $\left|f_{n}(Q)-\phi(n)\right| \leq D$ implies that the quotient $\left|\frac{f_{n}(Q)}{\phi(n)}\right|$ is also bounded above. Moreover, it is also bounded away from zero for sufficiently large values of $n$. Therefore, for all $Q \subset E_{n}$,

$$
\frac{1}{D} \leq\left|\frac{f_{k_{n}}(Q)}{\phi\left(k_{n}\right)}\right| \leq D
$$


The nested sets $\left\{E_{n}\right\}$ defined this way satisfy the requirements of Lemma 3.3. so the Hausdorff dimension of the set $E=\bigcap_{n} E_{n}$ is

$$
\operatorname{dim}_{\mathrm{H}}(E) \geq d-C(N, \epsilon)
$$

with $\lim _{N \rightarrow \infty} C(N, \epsilon)=0$.

According to Theorem 3 in Sullivan's paper [17]

$$
\frac{1}{C} \leq\left|\frac{U(z)}{\operatorname{dist}\left(z, z_{0}\right)}\right| \leq C,
$$

and Lemma 4.2 states that $\left|U(z)-f_{Q_{z}}\right| \leq A$, which we can combine with the inequality (6.7), $\frac{1}{D} \leq\left|\frac{f_{n}(Q)}{\phi(n)}\right| \leq D$, to get

$$
\frac{1}{C^{\prime}} \leq\left|\frac{\operatorname{dist}\left(\gamma(n), z_{0}\right)}{\phi(n)}\right| \leq C^{\prime} .
$$

This shows that $\operatorname{dim}_{\mathrm{H}}\left(\Lambda_{\phi}\right)=d$.

Choosing $\phi(t)=t$ the set $\Lambda_{\phi}$ determines the deep points, and using Theorem 6.1 we get the following corollary:

Corollary 6.3. If $G$ is a non-compact, geometrically infinite and topologically tame Kleinian group, $M=\mathbb{B} / G$ has injectivity radius bounded away from zero, and there exists a Green's function on $M$, then the deep points have dimension 2.

Similarly in the Fuchsian case:

Corollary 6.4. If $G$ is a geometrically infinite Fuchsian group so that there exists a Green's function on $M=\mathbb{B} / G$ and the injectivity radius is bounded away from zero as well as bounded from above, then the deep points have dimension 1.

The dimension of escaping points has been studied, and recently J. L. Fernández and M. V. Melián (in [9]) showed that the escaping geodesics on a finitely generated divergence type Riemann surface have dimension 1. Deep points form a smaller set, but perhaps one could derive a similar theorem for an intermediate set, for $\Lambda_{\phi}$ as defined in Section 1 with some function $\phi$.

\section{ACKNOWLEDGEMENT}

The author would like to give special thanks to her advisor, Christopher J. Bishop, for the many helpful consultations and for his useful comments and suggestions on the previous drafts of this work.

\section{REFERENCES}

[1] I. Agol, Tameness of Hyperbolic 3-manifolds, preprint, available at arXiv:math.GT/0405568, 2004.

[2] L. V. Ahlfors, Möbius transformations in several dimensions, Lecture Notes, School of Mathematics, University of Minnesota, Minneapolis, 1981. MR725161 (84m:30028)

[3] A. F. Beardon, The Geometry of Discrete Groups, Springer-Verlag, New York, 1983. MR698777 (85d:22026)

[4] C. J. Bishop and P. W. Jones, The law of the iterated logarithm for Kleinian groups, Contemp. Math. 211 (1997), 17-50. MR1476980 (98j:30051)

[5] C. J. Bishop and Y. Peres, Hausdorff dimension and Fractal Sets, to appear.

[6] D. Calegari and D. Gabai, Shrinkwrapping and the taming of hyperbolic 3-manifolds, J. Amer. Math. Soc. 19 (2006), 385-446. MR2188131 (2006g:57030) 
[7] R. D. Canary, Ends of hyperbolic 3-manifolds, J. Amer. Math. Soc. 6 (1993), 1-35. MR.1166330 (93e:57019)

[8] S.-Y. A. Chang, J. M. Wilson, and T. Wolff, Some weighted norm inequalities concerning the Schrödinger operators, Comment. Math. Helv. 60 (1985), 217-246. MR.800004 (87d:42027)

[9] J. L. Fernández and M. V. Melián, Escaping geodesics of Riemann surfaces, Acta Math. 187 (2001), 213-236. MR1879849 (2003d:30043)

[10] Z. Gönye, The Dimension of Escaping Points, Ph.D. thesis, State University of New York at Stony Brook, 2001.

[11] P. Hall and C. C. Heyde, Martingale Limit Theory and Its Application, Academic Press, New York, 1980. MR624435(83a:60001)

[12] G. J. Hungerford, Boundaries of smooth sets and singular sets of Blaschke products in the little Bloch space, Master's thesis, California Institute of Technology, 1988.

[13] B. Maskit, Kleinian Groups, Springer-Verlag, New York, 1988. MR959135 (90a:30132)

[14] C. T. McMullen, Renormalization and 3-Manifolds which Fiber over the Circle, Annals of Mathematics Studies, vol. 142, Princeton University Press, Princeton, 1996. MR 1401347 (97f:57022)

[15] P. J. Nicholls, The Ergodic Theory of Discrete Groups, Cambridge University Press, Cambridge, 1989. MR1041575 (91i:58104)

[16] S. Rohde, The boundary behavior of Bloch functions, J. London Math. Soc. (2) 48 (1993), no. 3, 488-499. MR 1241783 (94k:30083)

[17] D. Sullivan, Growth of positive harmonic functions and Kleinian group limit sets of zero planar measure and Hausdorff dimension two, Geometry Symposium 504 (1980), 127-144. MR655423 (83h:53054)

Department of Mathematics, State University of New York at Stony Brook, Stony Brook, New York 11794

Current address: Department of Mathematics, University of West Hungary, Szombathely, H9700, Hungary

E-mail address: zgonye@ttmk.nyme.hu 